\title{
Are Routine Jobs Moving South? \\ Evidence from Changes in the Occupational Structure of Employment in the U.S. and Mexico
}

Matías Cortés (York University)

Diego M. Morris (Nottingham Trent University)

April 2019 


\section{Motivation}

- Falling share of employment in routine task-intensive, middle-wage jobs

- Widely documented across developed countries (Goos, Manning \& Salomons, 2009; Acemoglu \& Autor, 2011)

- Potential driving forces:

(1) Falling trade/offshoring costs: e.g. Antras, Garicano \& Rossi-Hansberg (2006), Egger, Kreickemeier, Moser \& Wrona (2016)

(2) Routine-replacing technological change: e.g. Autor, Levy \& Murnane (2003), Goos \& Manning (2007)

- These two driving forces have very different implications in terms of patterns that should be observed in developing countries 


\section{Motivation}

(1) Falling trade/offshoring costs:

- Routine jobs moving South

- Should observe an increase in routine employment in countries such as Mexico

- "Our jobs are fleeing the country, they are going to Mexico, and many other countries" (Donald Trump, 2016)

(2) Routine-replacing technological change:

- Routine jobs disappearing everywhere

- Should observe a decrease in routine employment in countries such as Mexico 


\section{Our Paper}

- Contrast changes in occupational structure of employment in the U.S. and Mexico

- Use detailed job categories based on matching of occupational codes (job titles)

- Compare evolution of employment across common occupational categories, not along the occupational wage distribution

- Routine-intensive jobs need not be middle-wage jobs in Mexico

- Determine whether routine-intensive occupations are growing or shrinking in Mexico 


\section{Preview of Findings}

- Employment share patterns strongly correlated across the two countries

- In particular, routine manual jobs are declining in both countries

$\Rightarrow$ No support for hypothesis that these jobs have moved from the U.S. to Mexico

- Common shocks, e.g. the development of routine-replacing automation technologies, a more likely driver of the decline 


\section{Contributions to the Literature}

- Polarization Literature:

- So far, limited evidence on de-routinization outside of high-income countries [exceptions: World Bank (2016), Ariza \& Raymond Bara (2018), Reijnders \& de Vries (2018)]

- New evidence for Mexico; much finer level of detail; US-Mexico comparison

- Offshoring Literature:

- So far, mainly focused on impacts on skill premium [e.g. Goldberg \& Pavcnik (2007), Autor, Dorn \& Hanson (2013), Acemoglu, Gancia \& Zilibotti (2015), Burstein \& Vogel (2017), Hummels, Munch \& Xiang (2018)]

- Alternative approach to explore basic underlying idea that certain jobs are moving to developing countries due to falling trade/offshoring costs

- Impacts of Trade on Mexican Labor Market: e.g. lacovone, Rauch \& Winters (2013), Utar \& Torres Ruiz (2013), Mendez (2015) 


\section{Data}

National Labor Force Survey micro-data for both countries

\section{Mexico:}

- Encuesta Nacional de Empleo (ENE); Encuesta Nacional de Ocupación y Empleo (ENOE)

- Quarterly frequency; 2000-2015

- $\sim 594,000$ obs per year

\section{United States:}

- Current Population Survey (CPS)

- Monthly frequency

- 730,000 obs per year 


\section{Sample Restrictions}

- Civilian workers aged 16-65

- Non-missing information on current occupation

- Exclude workers in agriculture and farming occupations

- Mexican data includes both formal and informal sector workers 
Table: Descriptive Statistics for Employed Workers

\begin{tabular}{l|cc|cc}
\hline \hline & \multicolumn{3}{c}{ U.S. } & \multicolumn{2}{c}{ Mexico } \\
\hline & 2000 & 2015 & 2000 & 2015 \\
\hline & & & & \\
Average Age & 38.56 & 40.74 & 34.68 & 37.89 \\
Fraction Female & 46.90 & 47.20 & 37.36 & 41.21 \\
Average Real Wage (2009 USD) & 20.46 & 22.43 & 2.21 & 2.05 \\
Manufacturing Share of Emp & 15.11 & 10.49 & 23.69 & 18.69 \\
& & & & \\
Educational Composition: & & & & \\
$\quad$ Elementary Education or Less & 1.90 & 1.65 & 40.87 & 24.67 \\
$\quad$ Middle School & 9.22 & 5.68 & 25.33 & 32.60 \\
$\quad$ High School & 61.04 & 56.86 & 17.25 & 23.29 \\
$\quad$ College Education or Higher & 27.84 & 35.81 & 16.55 & 19.44 \\
& & & & \\
Nr of Observations (Unweighted) & 687,681 & 688,316 & 593,657 & 569,918 \\
\hline \hline
\end{tabular}


We match occupation codes across the two countries in order to compare the evolution of employment by occupation

Matching based on job titles: e.g., "accountants and auditors", "engineers and scientists"

100 occupational categories matched across the two countries. 


\section{Occupation Matching: Examples}

\begin{tabular}{|c|c|c|c|c|}
\hline occ_cm & Occupation Description & CMO Codes & SINCO Codes & occ1990dd Codes \\
\hline 1 & Accountants and auditors & 1172 & 2121 & 23 \\
\hline 2 & Actors, directors, and producers & 1413 & 2175 & 187 \\
\hline 3 & Architects & 1100 & 2263 & 43 \\
\hline 4 & Chemical engineers & 1104 & 2251 & 48 \\
\hline 5 & Chemists & 1120 & 2222 & 73 \\
\hline 6 & Civil engineers & 1101 & 2261 & 53 \\
\hline 7 & Dancers & 1414 & 2174 & 193 \\
\hline 8 & Dentists & 1132 & 2413 & 85,204 \\
\hline 9 & $\begin{array}{l}\text { Economists, market and survey } \\
\text { researchers }\end{array}$ & 1170 & 2123 & 166 \\
\hline 10 & Electrical engineers & 1105 & 2241,2642 & 55 \\
\hline 11 & $\begin{array}{l}\text { Insurance adjusters, examiners, } \\
\text { and investigators }\end{array}$ & 7134 & 4222 & $24,253,375$ \\
\hline 12 & $\begin{array}{l}\text { Lawyers/Legal assistants and } \\
\text { paralegals }\end{array}$ & 1160 & 2135 & 178,234 \\
\hline
\end{tabular}




\section{Occupation Matching: Examples}

\begin{tabular}{|c|c|c|c|c|}
\hline occ_cm & Occupation Description & CMO Codes & SINCO Codes & occ1990dd Codes \\
\hline 25 & Other type of art performer & $\begin{array}{l}1400,1419,1423,1429 \\
1430,1431,1432\end{array}$ & $\begin{array}{l}2151,2162,2164,2541, \\
2543,2544,2551,2655, \\
2712\end{array}$ & $\begin{array}{l}165,183,184,185,217 \\
645,649,684\end{array}$ \\
\hline 26 & Engineers and scientists & $\begin{array}{l}1106,1109,1110,1111, \\
1129,1140,1141,1149, \\
1150,1152,1153,1168\end{array}$ & $\begin{array}{l}2134,2211,2212,2221 \\
2223,2231,2242,2252, \\
2253,2421,2611,2612, \\
2613,2621,2622\end{array}$ & $\begin{array}{l}44,45,47,56,57,59,68, \\
69,74,76,77,78,79,83, \\
258,829,844\end{array}$ \\
\hline 27 & Other Teachers & $\begin{array}{l}1300,1310,1320,1330, \\
1331,1332,1340,1350, \\
1351,1352,1353,1354, \\
1359,1360,1361,1362, \\
1363,1364,1369,1390\end{array}$ & $\begin{array}{l}2321,2322,2331,2332, \\
2333,2334,2335,2339, \\
2341,2342,2343,2391, \\
2713,2714,2715\end{array}$ & $\begin{array}{l}154,155,156,157,158 \\
159,163,387\end{array}$ \\
\hline
\end{tabular}




\section{Grouping of Occupations}

Occupation Group

Occupation Codes (occ_cm)

Non-Routine Cognitive (NRC)

Management Related Occupations

Professional Specialty Occupations

Technicians and Related Support Occupations

$$
\begin{gathered}
1-27,30-32 \\
33-43,89-90 \\
44
\end{gathered}
$$

\section{Routine Cognitive (RC)}

Sales Occupations

Administrative Support Occupations

$$
\begin{aligned}
& 45-52 \\
& 53-61
\end{aligned}
$$

\section{Routine Manual (RM)}

Mechanics and Repairers

Production and Construction Related Occupations Machine Operators, Assemblers, and Inspectors

Transportation and Material Moving Occupations

$$
\begin{gathered}
62-64,74 \\
28,29,65-75 \\
76-85 \\
86
\end{gathered}
$$

\section{Non-Routine Manual (NRM)}

Housekeeping and Cleaning Occupations

Protective Service Occupations

87,88

91

Other Service Occupations 


\section{Employment Share Changes: US}

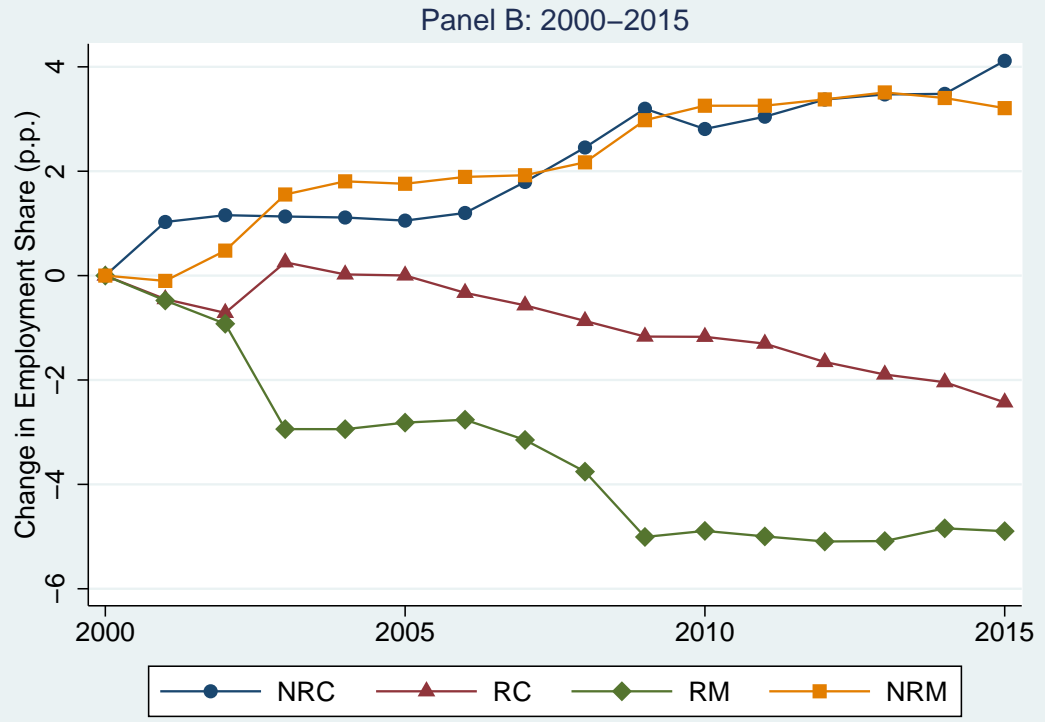




\section{Employment Share Changes: Mexico}

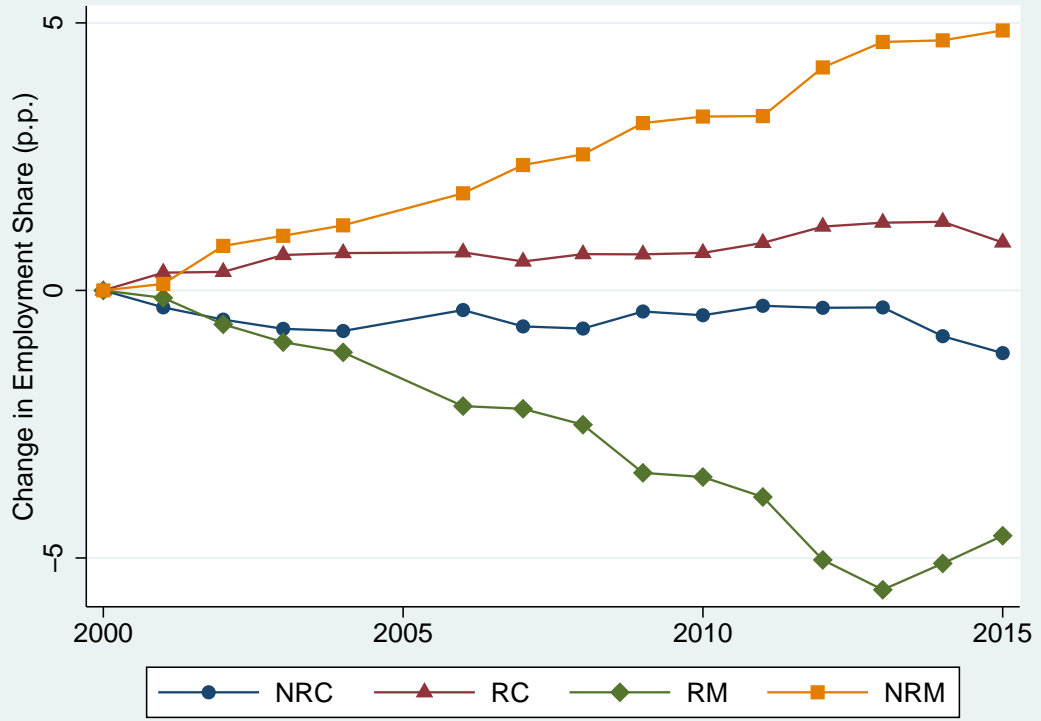




\section{Employment Share Changes: Mexico, 2000-2015}

Non Routine Cognitive

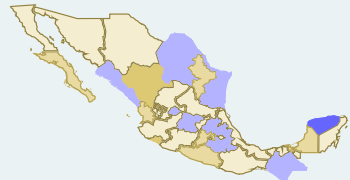

Routine Manual

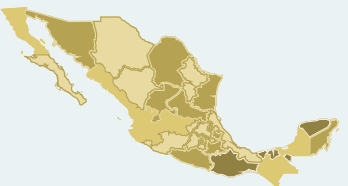

Routine Cognitive

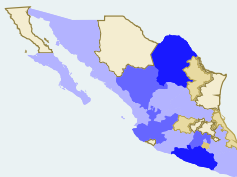

Non Routine Manual

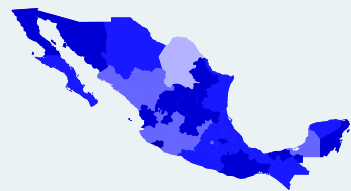

Change in Employment Share (p.p change)

\begin{tabular}{|l|}
$8-10$ \\
$6-8$ \\
$4-6$ \\
$2-4$ \\
$0-2$ \\
$-2-0$ \\
$-4--2$ \\
$-6--4$ \\
$-8--6$ \\
$-10--8$
\end{tabular}




\section{Employment Share Changes: US, 2003-2011}

Panel A: United States

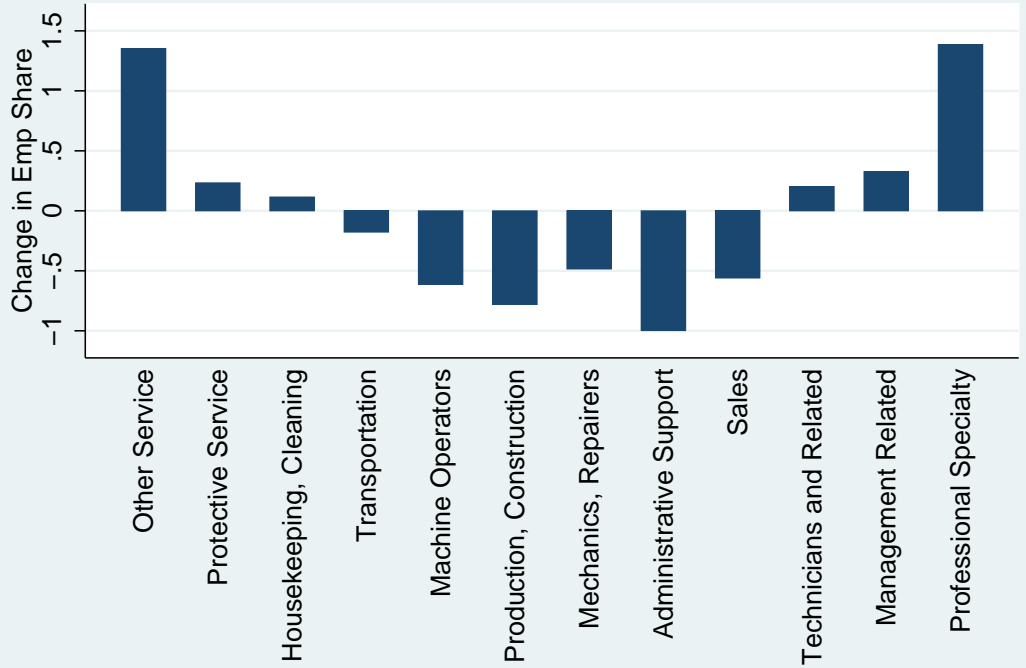




\section{Employment Share Changes: Mexico, 2003-2011}

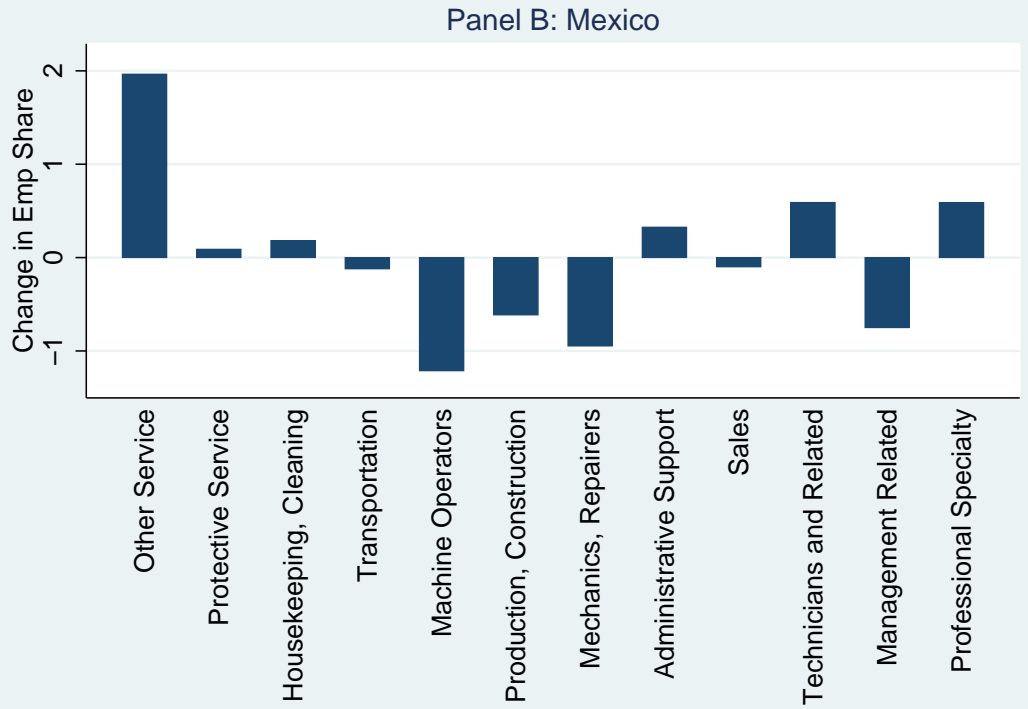




\section{Patterns Across Detailed Occupation Groups}




\section{Wages across 100 Detailed Occ Categ: US vs MX}

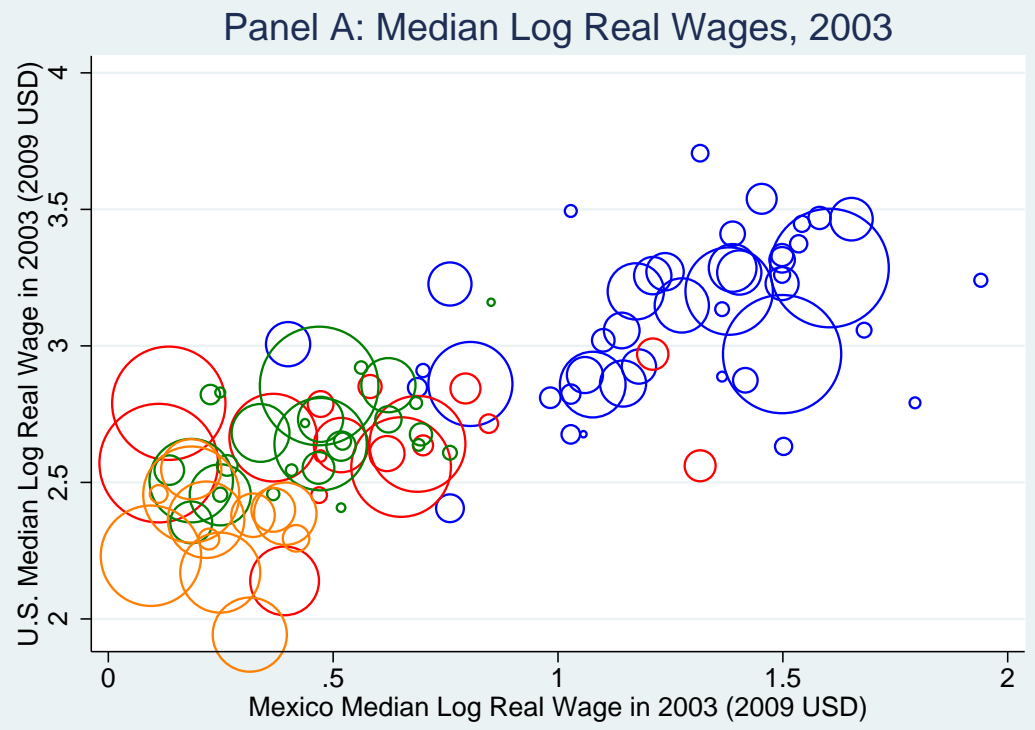




\section{Wage Ranking: US vs MX}

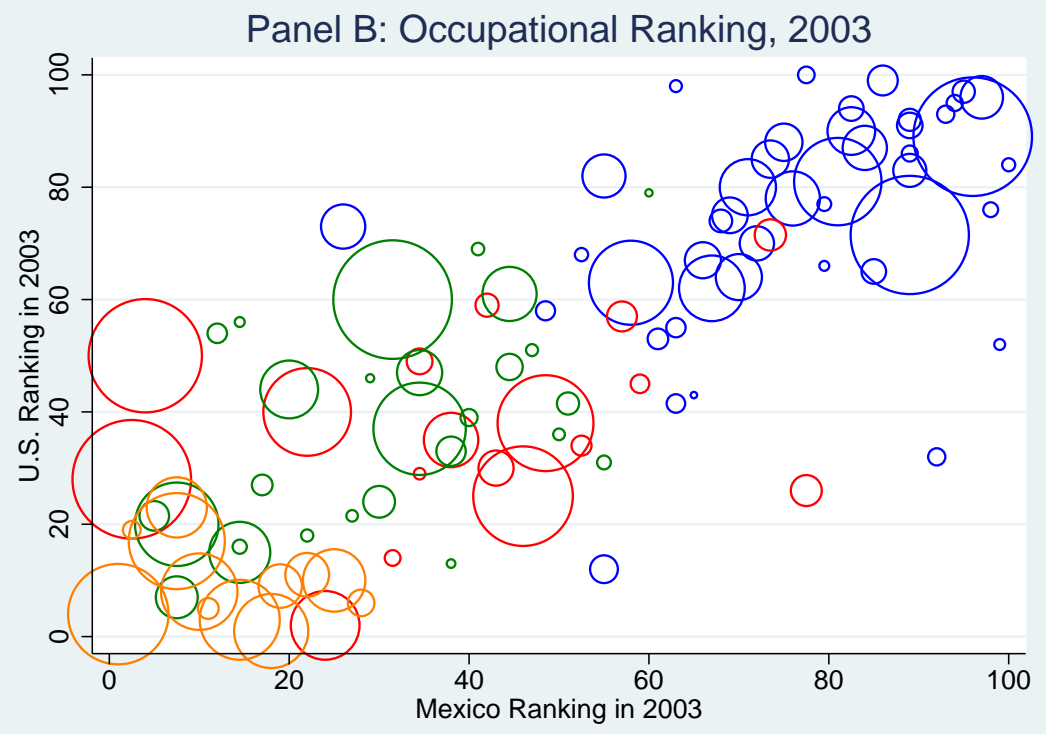




\section{Main Results: Emp Share Chgs, 2003-11, US vs MX}

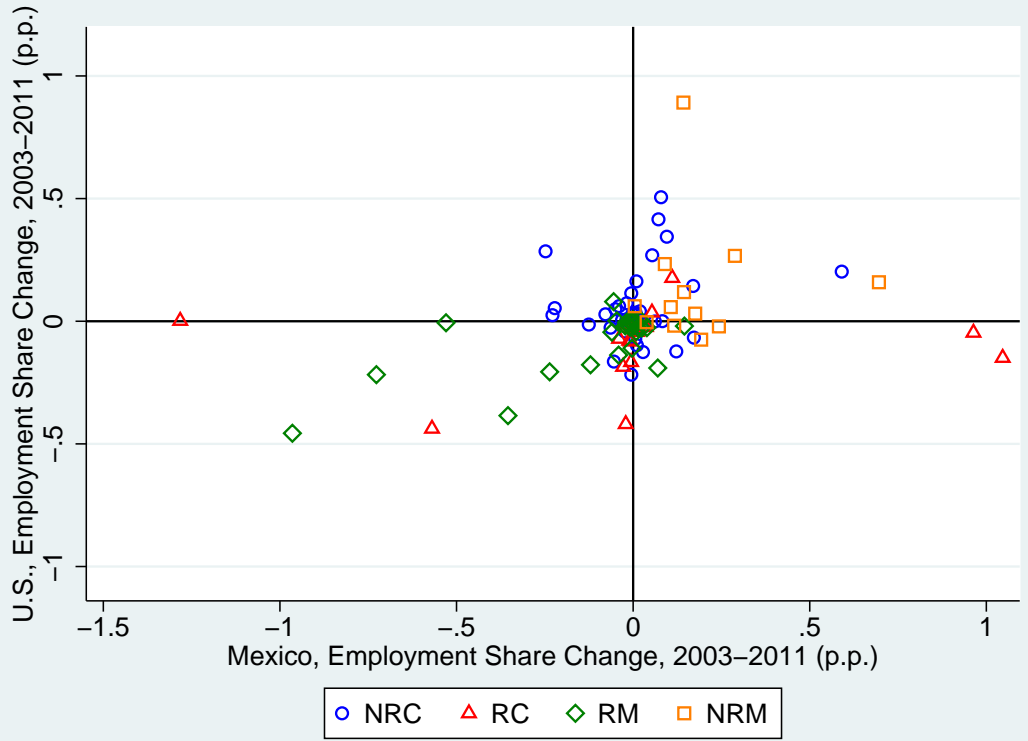




\section{Main Results: Emp Share Chgs, 2003-11, US vs MX}

Table: Occupations with largest declines in emp shares in the U.S., 2003-11

\begin{tabular}{l|c|cc}
\hline \hline & & \multicolumn{2}{|c}{ Chg in Emp Share (p.p.) } \\
& Group & U.S. & Mexico \\
\hline Production related occupations & RM & -0.457 & -0.965 \\
Secretary & RC & -0.438 & -0.569 \\
Merchant at commercial establishment & RC & -0.419 & -0.021 \\
Other machine operators n.e.c & RM & -0.385 & -0.354 \\
Winding and twisting textile and apparel operatives & RM & -0.218 & -0.727 \\
Construction inspectors & NRC & -0.218 & -0.005 \\
Carpenters & RM & -0.206 & -0.236 \\
Production, installation and maintenance laborers & RM & -0.191 & $\mathbf{0 . 0 7 0}$ \\
Data entry keyers & RC & -0.186 & -0.028 \\
Transportation and material moving occupations & RM & -0.178 & -0.121 \\
Typists & RC & -0.166 & -0.006 \\
Production supervisors or foremen & NRC & -0.164 & -0.054 \\
\hline \hline
\end{tabular}




\section{Main Results: Emp Share Chgs, 2003-11, US vs MX}

Table: Occupations with largest increases in emp shares in the U.S., 2003-11

\begin{tabular}{|c|c|c|c|}
\hline & \multirow[b]{2}{*}{ Group } & \multicolumn{2}{|c|}{ Chg in Emp Share (p.p. } \\
\hline & & U.S. & Mexico \\
\hline Other type of food cook, waiter & NRM & 0.159 & 0.696 \\
\hline Engineers and scientists & NRC & 0.163 & 0.009 \\
\hline Cashiers & $\mathrm{RC}$ & 0.177 & 0.111 \\
\hline Technicians & NRC & 0.202 & 0.591 \\
\hline Security professionals and related worker & NRM & 0.233 & 0.090 \\
\hline Food preparation or related worker & NRM & 0.267 & 0.288 \\
\hline Registered nurses & NRC & 0.269 & 0.054 \\
\hline Managers and administrators n.e.c. & NRC & 0.285 & -0.248 \\
\hline Other managers/coordinators n.e.c & NRC & 0.345 & 0.095 \\
\hline Other professionals n.e.c & NRC & 0.416 & 0.072 \\
\hline Other teachers & NRC & 0.506 & 0.079 \\
\hline Personal service occupations, n.e.c & NRM & 0.891 & 0.142 \\
\hline
\end{tabular}




\section{Lagged Changes?}

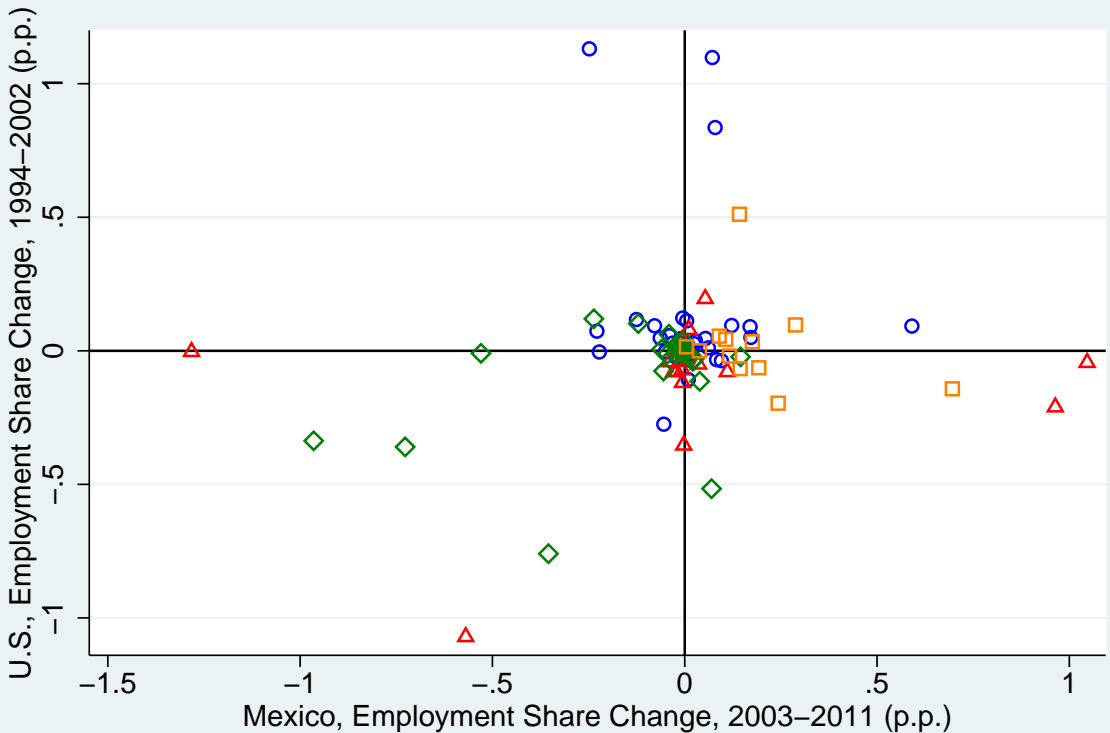

$\circ \mathrm{NRC} \Delta \mathrm{RC} \diamond \mathrm{RM} \square \mathrm{NRM}$ 
- We compare employment patterns across 100 detailed occupational categories in the U.S. and Mexico

- With few exceptions, routine manual jobs have been on the decline in both countries

$\Rightarrow$ No support for hypothesis that these jobs have moved from the U.S. to Mexico 


\section{Thank you!}

